\title{
A small angle neutron scattering and Mössbauer spectrometry study of magnetic structures in nanocrystalline $\mathrm{Ni}_{3} \mathrm{Fe}$
}

\author{
H. N. Frase and B. Fultz ${ }^{\mathrm{a})}$ \\ Division of Engineering and Applied Science, mail 138-78 California Institute of Technology, Pasadena, \\ California 91125
}

S. Spooner and J. L. Robertson

Solid State Division, Oak Ridge National Laboratory, Oak Ridge, Tennessee 37831

(Received 1 October 1998; accepted for publication 14 February 1999)

\begin{abstract}
Results are reported from small angle neutron scattering and Mössbauer spectrometry measurements on nanocrystalline $\mathrm{Ni}_{3} \mathrm{Fe}$. The nanocrystalline materials were prepared by mechanical attrition and studied in the as-milled state, after annealing at $265^{\circ} \mathrm{C}$ to relieve internal stress, and after annealing $600{ }^{\circ} \mathrm{C}$ to prepare a control sample comprising large crystals. The small angle neutron scattering (SANS) measurements were performed for a range of applied magnetic fields. Small differences were found in how the different samples reached magnetic saturation. From the SANS data obtained at magnetic saturation, we found little difference in the nuclear scattering of the as-milled material and the material annealed at $265^{\circ} \mathrm{C}$. Reductions in nuclear scattering and magnetic scattering were observed for the control sample, and this was interpreted as grain growth. The material annealed at $265^{\circ} \mathrm{C}$ also showed a reduction in magnetic SANS compared to the as-milled material. This was interpreted as an increase in magnetic moments of atoms at the grain boundaries after a low temperature annealing. Both Mössbauer spectroscopy and small angle neutron scattering showed an increase in the grain boundary magnetic moments after the $265^{\circ} \mathrm{C}$ annealing $\left(0.2\right.$ and $0.4 \mu_{B}$ /atom, respectively), even though there was little change in the grain boundary atomic density. (C) 1999 American Institute of Physics. [S0021-8979(99)07610-0]
\end{abstract}

\section{INTRODUCTION}

In the past decade there has been widespread interest in "nanostructured materials," defined here as materials having internal structures with spatial dimensions of $20 \mathrm{~nm}$ or smaller. Investigations of the magnetic properties of nanocrystalline materials have been stimulated by the soft magnetic properties of Finemet ${ }^{\circledR}$, which has a lower magnetic coercivity when nanocrystals are present than when the material is fully amorphous. In nanocrystalline materials, the sizes of the nanocrystals are often smaller than the expected widths of the magnetic domain walls. Magnetic domains of some sort are expected, however, because domains reduce the magnetic field energy external to the material. Previous work by small angle neutron scattering (SANS) identified small-scale magnetic structures in nanocrystalline materials having soft magnetic properties, including $\mathrm{Fe}^{1}$ and $\mathrm{Ni}^{2,3}$ (Some materials prepared by gas consolidation also contained nano-pores, which can make large contributions to the SANS. $)^{4-6}$ Magnetic microscopy has also been used to identify larger features of magnetic domain structures. ${ }^{7,8}$ The magnetic structures are expected to affect the shape of the $\mathrm{B}-\mathrm{H}$ magnetization curve, so a better understanding of the differences between magnetic structures of nanocrystalline materials and materials with large crystallites will help the understanding of magnetic properties of nanocrystalline materials.

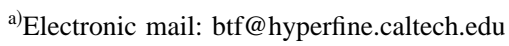

A high density of grain boundaries is one reason why nanocrystals have unconventional properties. ${ }^{9,10}$ From previous work, ${ }^{11}$ it is known that the grain boundaries of nanocrystalline $\mathrm{Ni}_{3} \mathrm{Fe}$ are about $0.5 \mathrm{~nm}$ wide. With $15 \mathrm{~nm}$ crystallites, face-centered-cubic (fcc) $\mathrm{Ni}_{3} \mathrm{Fe}$ will have a volume fraction of atoms at and near grain boundaries of about 0.1 . Although grain boundaries in nanocrystals are generally understood to be similar to grain boundaries in conventional large-grained materials, some differences have been suggested by previous work. For example, mechanical attrition may create grain boundaries having a higher energy than equilibrated high-angle grain boundaries. ${ }^{12}$ Although still controversial, other studies indicate that the grain boundary atomic structures of nanocrystals differ from those in conventional polycrystals, and may change upon annealing or aging. ${ }^{13-15}$ Different methods of synthesis may produce different types of grain boundaries. Mechanically attrited nanocrystals may have grain boundaries inherently different from those in nanocrystals prepared by nondeformation processes such as gas condensation. Considering the large volume fraction of grain boundaries in a nanocrystalline material, differences in the local atomic structure of grain boundaries could affect macroscopic properties such as mechanical deformation and atomic diffusivities. ${ }^{16-18}$

We report results from a study on the structure of grain boundaries and magnetic domains in the soft magnetic material $\mathrm{Ni}_{3} \mathrm{Fe}$. Powders were prepared by mechanical attrition, and were studied in three states: as-milled nanocrystals, nanocrystals after a stress relief treatment, and as a control 
sample comprised of large grains. We measured the magnetic and nuclear SANS from these different materials under a wide range of applied magnetic fields to investigate differences in their magnetic structure as they reached magnetic saturation. We found little difference in the saturation behavior of the three samples of $\mathrm{Ni}_{3} \mathrm{Fe}$, indicating that any differences in magnetic structures caused by domain formation occur on a spatial scale of $100 \mathrm{~nm}$ or larger. We present evidence from magnetic SANS and hyperfine magnetic field distributions obtained from Mössbauer spectrometry that a low temperature annealing causes a measurable increase in the magnetic moment per atom in the grain boundary regions. We attribute the effect of an annealing at $265^{\circ} \mathrm{C}$ to relaxation in local atomic configurations. These relaxations in atomic configurations are small, however, since the nuclear SANS indicates that the atomic density of the grain boundary region is not altered significantly by low temperature annealing.

\section{EXPERIMENT}

Nanocrystalline $\mathrm{Ni}_{3} \mathrm{Fe}$ powders were prepared by mechanical attrition in a SPEX $8000 \mathrm{mixer} / \mathrm{mill}$. Stoichiometric amounts of $\mathrm{Ni}$ and $\mathrm{Fe}$ powders and $7.5 \mathrm{ml}$ of ethanol were placed in hardened steel vials with steel balls, and were milled for $12 \mathrm{~h}$ with a ball-to-powder weight ratio of 2:1. Afterwards, a portion of the as-milled powder was annealed for $90 \mathrm{~min}$ at $265^{\circ} \mathrm{C}$ to create a material having a crystal size distribution similar to the as-milled sample, but with reduced internal stress. A separate annealing at $600{ }^{\circ} \mathrm{C}$ was performed on another portion of the as-milled powder to prepare a material with large crystals that would serve as a control sample. A few grams of the as-milled nanocrystalline powder were heated to $400^{\circ} \mathrm{C}$ and analyzed for hydrogen, oxygen, and nitrogen by a Hewlett-Packard 5890 gas chromatograph equipped with a thermal conductivity detector. The detected gas impurities were 0.003 wt $\%$ nitrogen, 0.001 wt \% carbon, and $0.003 \mathrm{wt} \%$ oxygen. No evolution of hydrogen was detected. Detection limit for hydrogen was better than $0.004 \mathrm{wt} \%$. We do not expect any significant neutron scattering from hydrogen.

Transmission electron microscopy (TEM) was performed with a Philips EM420 microscope operated at 120 $\mathrm{kV}$. Dark field micrographs were used to determine the size distribution of the nanocrystals. Figure 1 shows TEM micrographs of the as-milled and annealed materials. The internal structure of the powders can be described as a threedimensional mosaic of nanocrystals separated by grain boundaries. Size distributions of the nanocrystalline powders were obtained from the micrographs by drawing lines at random on the micrographs, measuring the length of intersection between the line and each nanocrystal, then converting the distribution of lengths to nanocrystal size distribution. ${ }^{19,20}$ There was little change in the nanocrystal size distribution after annealing at $265^{\circ} \mathrm{C}$, although annealing at $600{ }^{\circ} \mathrm{C}$ caused the grains to grow to sizes inappropriate for quantitative measurement by TEM.

Mechanical attrition produces nanocrystals that are strained and have a lognormal size distribution. X-ray dif-

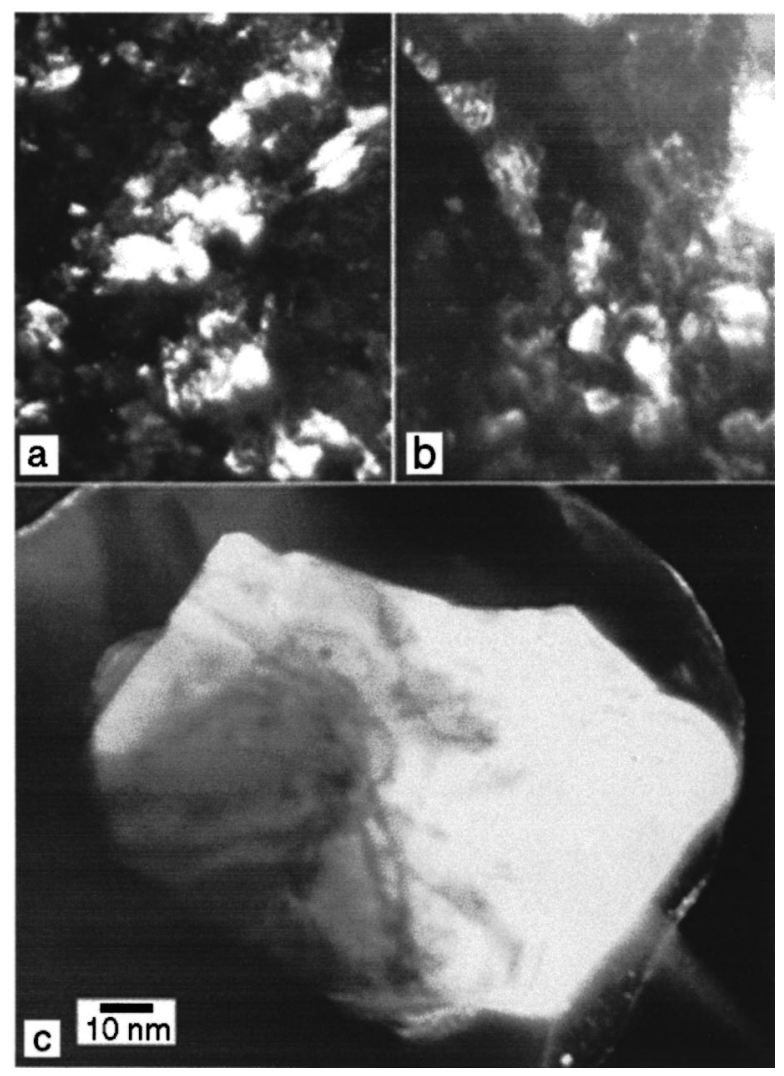

FIG. 1. TEM dark field images from (111) fec diffraction of $\mathrm{Ni}_{3} \mathrm{Fe}$-powders (a) as-milled, (b) $265^{\circ} \mathrm{C}$ annealed, and (c) $600{ }^{\circ} \mathrm{C}$ annealed.

fraction patterns, analyzed by the Williamson-Hall method, ${ }^{21}$ showed a reduction in root-mean-squared strain, $\left\langle\sqrt{\epsilon^{2}}\right\rangle$, from 0.10 to 0.07 after annealing at $265^{\circ} \mathrm{C}$. This Williamson-Hall analysis also showed that the average nanocrystal size increased from $15 \mathrm{~nm}$ in the as-milled material to $20 \mathrm{~nm}$ after annealing at $265^{\circ} \mathrm{C}$.

Scanning electron microscopy (SEM) images of the materials were obtained with a Camscan Series II scanning electron microscope. From the secondary electron SEM images such as shown in Fig. 2, it can be seen that the particles are $\sim 20 \mu \mathrm{m}$ in diameter. The particles do not have smooth surfaces, and instead have many cracks and appear "flaky" (Fig. 2). These "large-scale" features of the particles were found in all $\mathrm{Ni}_{3} \mathrm{Fe}$ powders, regardless of thermal annealing.

Mössbauer spectra were obtained at room temperature with a constant acceleration spectrometer in transmission geometry. The $\gamma$-rays were provided by a radiation source of ${ }^{57} \mathrm{Co}$ in a Rh matrix. Figure 3 shows the Mössbauer spectra of as-milled $\mathrm{Ni}_{3} \mathrm{Fe}$ materials and the materials after annealing at 265 and $600^{\circ} \mathrm{C}$. Also indicated in Fig. 3 are the peaks from the $\alpha$-Fe impurities present in the samples. These Fe impurities are from unincorporated ball fragments, which chip off the ball during the milling process.

The SANS measurements were performed at the $30 \mathrm{~m}$ SANS facility at the Oak Ridge National Laboratory, using a range of $Q$ from 0.075 to $0.6553 \mathrm{~nm}^{-1}$. The powder samples were placed in $2.54 \mathrm{~cm}$ diameter by $6 \mathrm{~mm}$ thick quartz holders. Cadmium masks were used to reduce background scattering from the quartz holder. The sample holder was posi- 


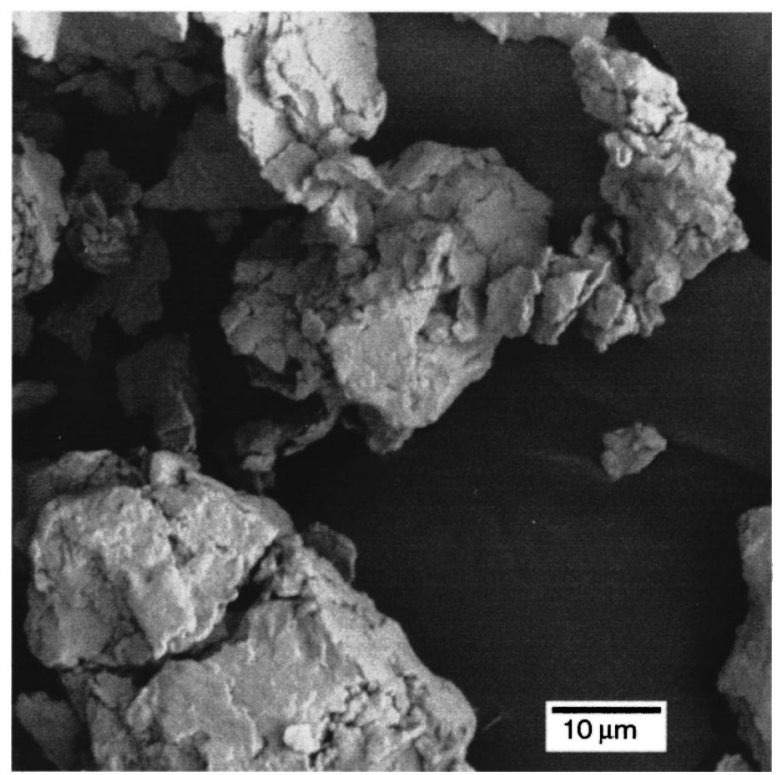

FIG. 2. SEM image of $\mathrm{Ni}_{3} \mathrm{Fe}$ powder particles as-milled with a 2:1 ball-topowder weight ratio.

tioned between the poles of an electromagnet. Scattering intensities were acquired when magnetic fields of 5-8000 Oe were applied to the sample perpendicular to the neutron beam. These data were first corrected for background. Using a Porosil A standard scatterer for calibration, the scattering intensity was then calibrated so the data were converted to an absolute differential cross section per unit volume. Figure 4 shows two-dimensional scattering contours for the three samples.

The $8 \mathrm{kOe}$ applied magnetic field allowed separation of the nuclear and magnetic scattering profiles. When a magnetic field is applied, the nuclear scattering, $I_{n}$, is isotropic, but the magnetic scattering, $I_{m}$, is maximized perpendicular to the field as

$$
I_{\text {total }}=I_{n}+I_{m} \sin ^{2}(\phi),
$$

where $\phi$ is the angle between the scattering and magnetization vectors. ${ }^{22}$ Parallel to the applied field the scattering is

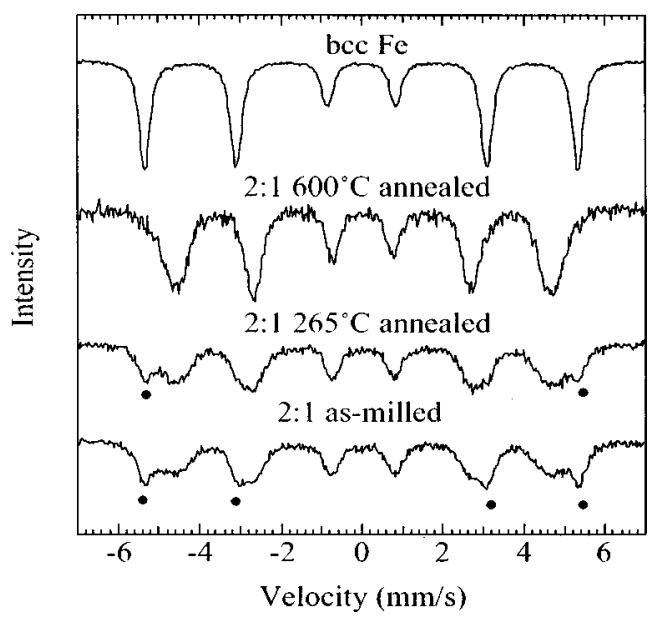

FIG. 3. Room temperature Mössbauer spectra of $\mathrm{Ni}_{3} \mathrm{Fe}$ samples (bcc $\mathrm{Fe}$ impurity peaks indicated by dots).

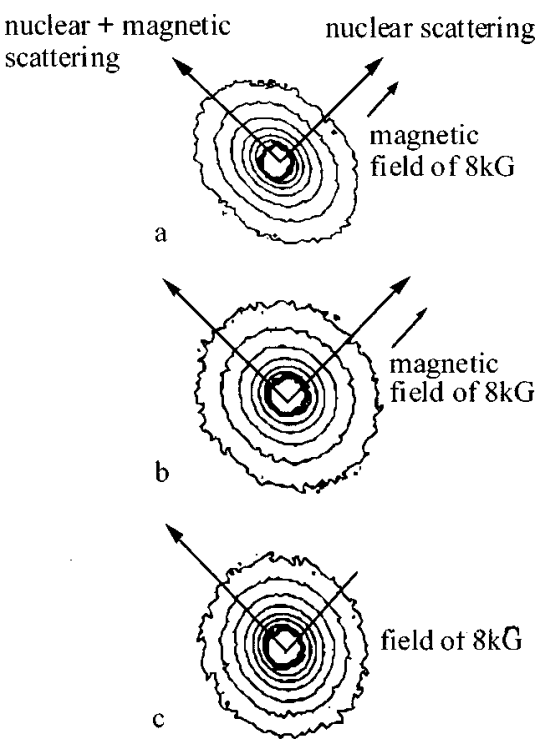

FIG. 4. Scattering contours in 8 kOe applied magnetic field showing directions for the scattering profiles of (a) as-milled, (b) $265^{\circ} \mathrm{C}$ annealed, and (c) $600{ }^{\circ} \mathrm{C}$ annealed powders.

only $I_{n}$, while perpendicular to the applied field it is $I_{n}$ $+I_{m}$. By subtracting the nuclear scattering profile from the nuclear plus magnetic scattering profile, the magnetic scattering profile was obtained.

\section{HYPERFINE MAGNETIC FIELDS}

Hyperfine magnetic field (HMF) distributions were extracted from the experimental Mössbauer spectra by the method of Le Caër and Dubois. ${ }^{23}$ The distributions were corrected for the presence of a $330 \mathrm{kOe}$ peak from $\mathrm{Fe}$ contamination by ball fragments, and the resulting HMF distributions were normalized. They are shown in Fig. 5. The prominent peak for the control sample annealed at $600{ }^{\circ} \mathrm{C}$ is centered on $287 \mathrm{kOe}$, typical of large-grained, chemically disordered $\mathrm{Ni}_{3} \mathrm{Fe}^{24-26}$ We expect the HMFs of the ${ }^{57} \mathrm{Fe}$ atoms inside the nanocrystals to be similar to those of large-grained disor-

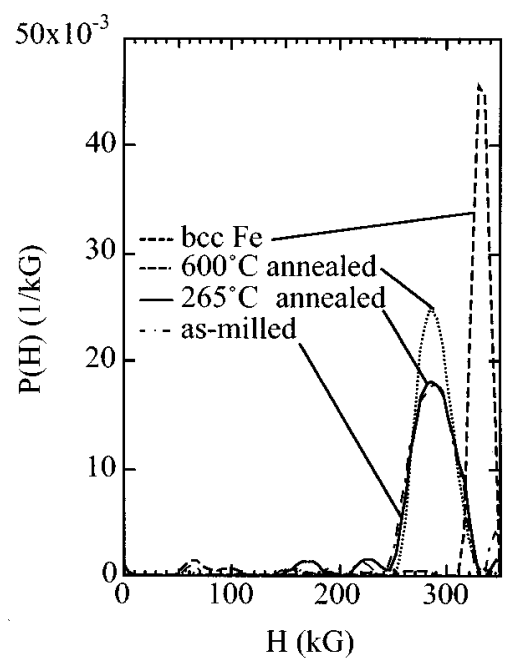

FIG. 5. Normalized HMF distribution with contribution from the Fe impurity removed. 
dered $\mathrm{Ni}_{3} \mathrm{Fe}$. The intensity in the HMF distribution below $242 \mathrm{kOe}$ has been shown to originate with ${ }^{57} \mathrm{Fe}$ atoms in and adjacent to grain boundaries. ${ }^{9,27}$ The detailed structure of this part of the HMF distribution is not unique, and the peaks in this range of HMF can be altered by different choices of fitting parameters such as instrument linewidth. The areas are reliable, however, as is the first moment. By calculating the area under the grain boundary component of the HMF distribution, the fraction of atoms at and near grain boundaries in the as-milled materials was estimated as 0.08 .

The low field components of the HMF distribution were used to estimate the magnetic moment of the grain boundary. The origin of the HMF in body-centered-cubic (bcc) Fe alloys is understood in detail, ${ }^{28}$ but this is not so for fcc Fe alloys. In analogy to bcc Fe alloys, however, we expect one contribution of the HMF to be the core polarization of inner $s$ electrons by the unpaired $3 d$ electrons at the ${ }^{57} \mathrm{Fe}$ atom. This contribution will be proportional to the magnetic moment of the ${ }^{57} \mathrm{Fe}$ atom itself. A second contribution to the HMF is the magnetic polarization of the $4 s$ conduction electrons by the magnetic moments at atoms near the ${ }^{57} \mathrm{Fe}$ atom. For disordered solid solutions, this contribution should be proportional to the average magnetic moment in the alloy. The ${ }^{57} \mathrm{Fe}$ HMF distribution of fcc $\mathrm{Fe}-\mathrm{Ni}$ alloys should therefore be interpretable with the following expression for the $\mathrm{HMF}$ at a ${ }^{57} \mathrm{Fe}$ atom, $H$

$$
H=\alpha \mu_{0}+\beta\langle\mu\rangle \text {. }
$$

Since the average magnetic moment of the alloy is

$$
\langle\mu\rangle=\frac{1}{N} \sum_{j}^{N} \mu_{j}
$$

we obtain:

$$
H=\alpha \mu_{0}+\frac{\beta}{N} \sum_{j}^{N} \mu_{j},
$$

where $\mu_{0}$ is the magnetic moment of the ${ }^{57} \mathrm{Fe}$ atom $\left(3.1 \mu_{B}\right),{ }^{29} \mu_{j}$ is the magnetic moment of the $j^{\text {th }}$ nearest neighbor $\left(0.65 \mu_{B}\right.$ if a $\mathrm{Ni}$ atom, $3.1 \mu_{B}$ if an $\mathrm{Fe}$ atom, and not strongly sensitive to the local chemistry). ${ }^{30}$ The HMF decreases upon ordering and decreases with $\mathrm{Ni}$ concentration, ${ }^{24,31,32}$ so $\alpha$ and $\beta$ are positive constants.

Since the first term of Eq. (4) changes only slightly with composition, the HMF should be less sensitive to the $\mathrm{Ni}$ concentration than the average magnetic moment, $\langle\mu\rangle$, and this is observed. ${ }^{25}$ A ratio of HMFs is therefore less sensitive to composition than a ratio of magnetic moments in the large-grained alloy. This is not necessarily true for a disordered structure such as a grain boundary. The mean HMFs in the grain boundaries of the as-milled sample $(140 \mathrm{kG})$ and after annealing at $265^{\circ} \mathrm{C}(180 \mathrm{kG})$ are lower than those of any fcc $\mathrm{Ni}-\mathrm{Fe}$ alloy. The HMFs of ${ }^{57} \mathrm{Fe}$ in the grain boundaries are evidently affected more strongly by the structural disorder than chemical disorder. It could be argued that the low HMF in the grain boundary region originates from a change in the coefficients $\alpha$ and $\beta$ in Eq. (2), as opposed to a change in the magnetic moments themselves. We do not expect the coefficient $\alpha$ to depend on the local atomic struc-

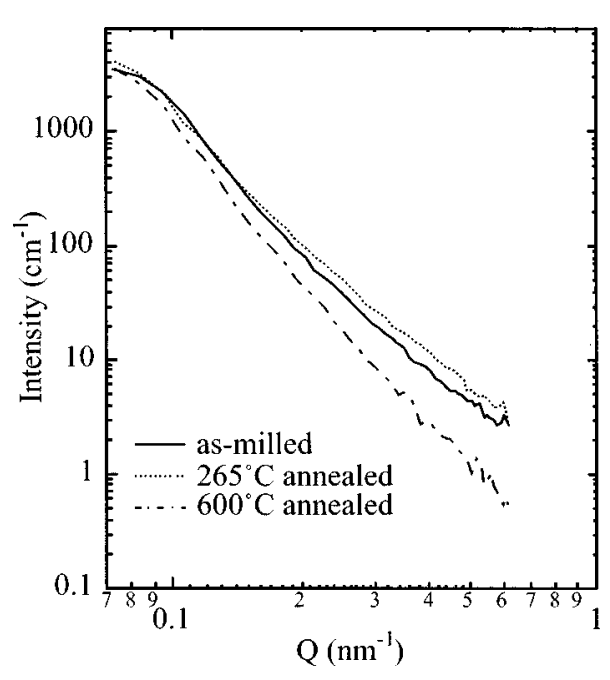

FIG. 6. Nuclear scattering profiles from the as-milled, $265^{\circ} \mathrm{C}$ annealed, and $600{ }^{\circ} \mathrm{C}$ annealed $\mathrm{Ni}_{3} \mathrm{Fe}$ powder.

ture, however, since it represents core polarization at the ${ }^{57} \mathrm{Fe}$ atom itself. The coefficient $\beta$ is much smaller than $\alpha$, indicative of the weak dependence of the ${ }^{57} \mathrm{Fe} \mathrm{HMF}$ on Ni concentration. Most of the large reduction in the HMF of grain boundary $\mathrm{Fe}$ atoms should, therefore, originate with a reduction of the Fe magnetic moments.

We obtain a lower bound on the magnetic moments of the atoms in the grain boundaries by taking the ratio of the average $\mathrm{HMF}$ of the grain boundary, $\mu_{\mathrm{GB}}$, to the HMF of large-grained $\mathrm{Fe}-\mathrm{Ni}(287 \mathrm{kG})$

$$
\mu_{\mathrm{GB}}=\mu_{\mathrm{XTL}} \frac{H_{\mathrm{GB}}}{287 \mathrm{kG}} \text {. }
$$

Using the magnetic moment of crystalline $\mathrm{Fe}-\mathrm{Ni}, \mu_{\mathrm{XTL}}$ $=1.2 \mu_{B} /$ atom, we obtain for the magnetic moments of the grain boundaries in as-milled material $\mu_{\mathrm{XTL}}=0.6 \mu_{B} /$ atom, and for the material annealed at $265^{\circ} \mathrm{C}, \mu_{B}=0.8 \mu_{B}$ /atom. We emphasize that these values are probably lower bounds for the magnetic moments of grain boundary atoms. The $\mathrm{Ni}$ magnetic moments are probably less affected by structural disorder than are magnetic moments at ${ }^{57} \mathrm{Fe}$ atoms, since the $3 d \uparrow$ states at $\mathrm{Ni}$ atoms are filled.

\section{SMALL ANGLE NEUTRON SCATTERING}

\section{A. Nuclear and magnetic scattering profiles}

Figure 6 shows the nuclear scattering profiles from the as-milled material and the material after annealing at 265 and $600{ }^{\circ} \mathrm{C}$. The nuclear scattering from the as-milled material and the material annealed at $265^{\circ} \mathrm{C}$ are similar. Both are more intense than the scattering from the sample annealed at $600{ }^{\circ} \mathrm{C}$. Larger differences were found for the magnetic scattering. Figure 7 shows that the material annealed at $600{ }^{\circ} \mathrm{C}$ had the smallest scattering intensity. Except for the smallest values of $Q$, the ratio of magnetic scattering for the materials annealed at 265 and $600^{\circ} \mathrm{C}$ was the same as the ratio of nuclear scattering from these two materials. This suggests that the differences in magnetic correlations from annealing at 265 versus $600{ }^{\circ} \mathrm{C}$ have the same origin as the differences 


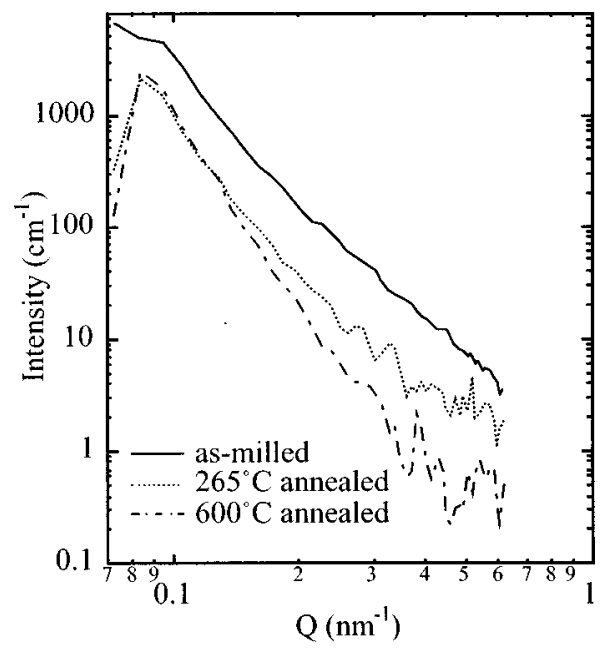

FIG. 7. Magnetic scattering profiles from the as-milled, $265^{\circ} \mathrm{C}$ annealed, and $600{ }^{\circ} \mathrm{C}$ annealed $\mathrm{Ni}_{3} \mathrm{Fe}$ powder in a $8 \mathrm{kG}$ applied magnetic field.

in atom density correlations from annealing at these two temperatures. We will argue that this difference originates from the scattering contrast difference between the nanocrystals and their grain boundaries.

SANS intensity profiles originate with several sources of scattering. The most important sources of scattering contrast are differences in the density of magnetic moments or atomic nuclei between the crystalline interior of the grains and the grain boundaries, the characteristic sizes of the grains and magnetic domains, and the morphologies of the grains and domains. The powder particles on the other hand (see Fig. 2) are too large to contribute significantly to the scattering intensity in the $Q$ range measured. The internal cracks, however, if sufficient in number, could contribute to the observed scattering. Nano-pores are common in samples made by compacting nanocrystals processed by gas condensation, and these nano-pores have led to problems in the interpretation of SANS data. We do not believe that nano-pores are present in our mechanically attrited samples, since the dense mosaic of nanocrystals is formed by plastic deformation of large crystals rather than by the compacting of individual nanocrystals. Figure 6 shows that there is little change in the nuclear scattering upon annealing at $265^{\circ} \mathrm{C}$. If nano-pores were present in the as-milled material, they do not change enough upon annealing at $265^{\circ} \mathrm{C}$ to affect significantly the total scattering intensity over the $Q$ range measured. Therefore, changes in nano-pores upon annealing cannot be responsible for the large difference in the magnetic scattering between the asmilled powder and the powder annealed at $265^{\circ} \mathrm{C}$, which is our most interesting result.

The magnetic scattering shows a large difference in intensity between the as-milled powder and the powder annealed at $265^{\circ} \mathrm{C}$. Although their similar nuclear scattering indicates similar atomic density correlations, the differences in magnetic scattering show that these two nanocrystalline materials have different magnetic density correlations. It is most likely that these changes in magnetic scattering originate with differences in magnetic moments in the grain boundaries. For the same microstructure, the magnetic scat- tering is proportional to the square of the difference in magnetic moments between the nanocrystals and grain boundaries. Since the scattering from the as-milled powders is larger, its grain boundaries must have a smaller average magnetic moment. The magnetic moments of atoms in the grain boundaries evidently become larger upon annealing at $265^{\circ} \mathrm{C}$. We suggest this originates with small changes in the local atomic structure in the grain boundaries. Previous work has shown that the grain boundaries of mechanically attrited nanocrystals have a broad distribution of interatomic distances with low atomic coordination numbers ${ }^{33}$ and that the severe nonequilibrium displacement of atoms in the grain boundaries results in an internal energy density higher than in conventional grain boundaries. ${ }^{12}$ It is plausible that upon annealing, some atoms in the grain boundaries make small movements into lower energy configurations. These changes do not affect the atomic density in the grain boundary in a significant way, but the nearest neighbor environments are altered enough to cause an increase in magnetic moment.

\section{B. Magnetic SANS of nanocrystals}

The intensity from a collection of independent scatterers with a size distribution, $P(R)$, is

$$
I(Q)=N P(R)(\Delta \rho)^{2} V^{2} F^{2}(Q R),
$$

where $N$ is the number density of scatterers, $\Delta \rho$ is the difference in either the nuclear or magnetic scattering amplitude between the scatterer and its matrix, $V$ is the volume of the particle, and $F(Q R)$ is the form factor of the scatterer. If the nuclear and magnetic scattering originate from the same microstructural features, (i.e., grain boundaries), the intensity of the magnetic scattering, $I_{m}(Q)$, will be proportional to the intensity of the nuclear scattering, $I_{n}(Q)$

$$
I_{m}(Q)=\frac{\Delta_{m} \rho_{m}}{\Delta_{n} \rho_{n}} I_{n}(Q) .
$$

The magnetic scattering length density, $\rho_{m}$, is equal to the magnetic moment of the material times a known constant, and the nuclear scattering length density, $\rho_{n}$, is the average atomic density times the average nuclear scattering length for nuclear coherent scattering. ${ }^{22}$ Both scattering length densities are constants for $\mathrm{Ni}_{3} \mathrm{Fe}$. The scattering factor contrast is provided by the fractional variation of scattering length densities between the scattering source and the surrounding matrix, which are $\Delta_{n}$ and $\Delta_{m}$ for nuclear and magnetic scattering. We expect the scattering from the as-milled powder and the powder annealed at $265^{\circ} \mathrm{C}$ to differ in the $\Delta_{m}$ factor. Evidently $\Delta_{m}$ decreases upon annealing due to an increase in magnetic moment density in the grain boundary. By varying $\Delta_{m}$ to fit the data, we can obtain a value for the average magnetization of the grain boundary region. To do so, we first subtracted the scattering factor profiles of the control sample from the scattering profiles of the nanocrystalline materials. We expect this subtraction will remove the scattering contributions from morphological features such as shown in Fig. 2, which were similar for all materials. The resulting nuclear and magnetic scattering intensities are shown in Fig. 8. For both the as-milled powder and the pow- 


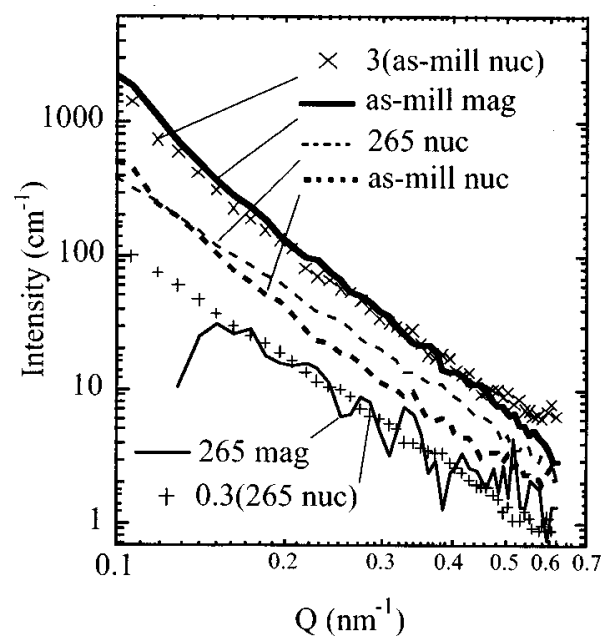

FIG. 8. Nuclear (nuc) and magnetic (mag) scattering profiles of the asmilled powders (as-mill) and the powders annealed at $265^{\circ} \mathrm{C}(265)$ after subtracting the scattering profiles of the control sample annealed at $600{ }^{\circ} \mathrm{C}$. Also shown are the nuclear scattering multiplied by the constant factors $(3.0$ and 0.4) used to fit the magnetic scattering.

der annealed at $265^{\circ} \mathrm{C}$, the magnetic scattering was fit by multiplying the appropriate nuclear scattering by a constant factor. Assuming that $\Delta_{n}$ is the same for both nanocrystalline samples, which is reasonable from the similar nuclear scattering intensity profiles, with Eq. (7) the fitting provided the $\Delta_{m}$ as a function of $\Delta_{n}$. We find $\Delta_{m}^{\mathrm{am}}=7.9 \Delta_{n}$ and $\Delta_{m}^{265}$ $=1.1 \Delta_{n}$. The superscripts refer to the as-milled powder and the powder annealed at $265^{\circ} \mathrm{C}$. The fractional variation of the magnetization in the material annealed at $265^{\circ} \mathrm{C}$ is approximately the same as its fractional variation of nuclear scattering length density. The magnetic contrast between the grain boundary and the matrix is much larger for the asmilled material.

Using the bulk $\mathrm{Ni}_{3} \mathrm{Fe}$ magnetization for the crystalline matrix $\left(1.2 \mu_{B} /\right.$ atom $)$, the grain boundary magnetic moments are

$$
\mu_{\mathrm{GB}}^{\mathrm{am}}=\left(1-7.9 \Delta_{n}\right) 1.2 \mu_{B} / \text { atom }
$$

and

$$
\mu_{\mathrm{GB}}^{265}=\left(1-1.1 \Delta_{n}\right) 1.2 \mu_{B} \text { /atom. }
$$

If atomic density of the grain boundaries were $95 \%$ that of the crystals, the grain boundary magnetic moments of the as-milled powder and the powder annealed at $265^{\circ} \mathrm{C}$ would be 0.7 and $1.1 \mu_{B} /$ atom, respectively.

\section{Radial distribution functions}

The nuclear and magnetic scattering profiles were converted into radial distribution functions (RDF), $G(R)$, by Fourier transformation of the intensity profiles, $I(Q)$

$$
G(R)=\frac{1}{4 \pi R} \int_{Q \min }^{Q \max } Q \sin (Q R) I(Q) d Q .
$$

To isolate features of the RDF associated with the nanocrystals themselves, the $I(Q)$ for the material annealed at $600^{\circ} \mathrm{C}$ was subtracted from the $I(Q)$ for the as-milled material and

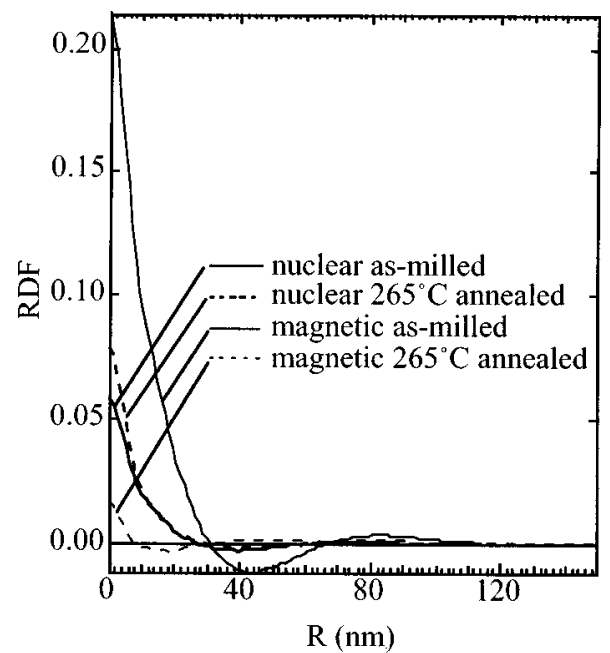

FIG. 9. Radial distribution functions for the as-milled and $265^{\circ} \mathrm{C}$ annealed powders after the scattering for the $600^{\circ} \mathrm{C}$ annealed sample was subtracted.

the $I(Q)$ of the material annealed at $265^{\circ} \mathrm{C}$. The resulting RDFs should relate only to the nanocrystals. They are shown in Fig. 9.

The two salient features of Fig. 9 are the similarity of the two RDFs for the nuclear scattering of the as-milled material and the material annealed at $265^{\circ} \mathrm{C}$, and the difference in their corresponding magnetic RDFs. The similarity of the nuclear curves supports our claim that the two samples have similar size distributions, number densities of scatterers, and grain boundary atomic densities. The large difference between the magnetic curves once again indicates that the magnetic moments of the grain boundaries are different. The first zero in the RDF is $\sim 30 \mathrm{~nm}$ for the two nuclear scattering profiles and for the magnetic scattering of the as-milled material. Since this is approximately the largest diameter of the nanocrystals, the scattering intensity at smaller $R$ is consistent with the size of the nanocrystals. (The oscillations of the RDFs after their first zero are end effects caused by the finite range of $Q$ available for Fourier transformation.) The RDF of the magnetic scattering from the material annealed at $265^{\circ} \mathrm{C}$ is relatively small at small values of $R$. This is consistent with an increase in the magnetic moment of the grain boundaries after annealing at $265^{\circ} \mathrm{C}$ and with a small difference in magnetic moment between the nanocrystals and grain boundaries.

From the RDF at $0 \mathrm{~nm}, G(0)$, it is possible to estimate the change in magnetic moments in grain boundaries after annealing at $265^{\circ} \mathrm{C}$. Equations (6) and (10) can be combined

$$
\begin{aligned}
G_{\text {nano }}(R)= & \frac{1}{4 \pi R} N P(R)(\Delta \rho)^{2} V^{2} \\
& \times \int_{Q \min }^{Q \max } Q \sin (Q R) F^{2}(Q R) d Q .
\end{aligned}
$$

Since the $G(R)$ for nuclear scattering was similar in shape to that for magnetic scattering, we assumed that each variable in Eq. (11) is the same for the as-milled and annealed at $265^{\circ} \mathrm{C}$ samples, except for the $\Delta \rho$ 's. This is the same assumption used for analyzing the magnetic SANS profile used 

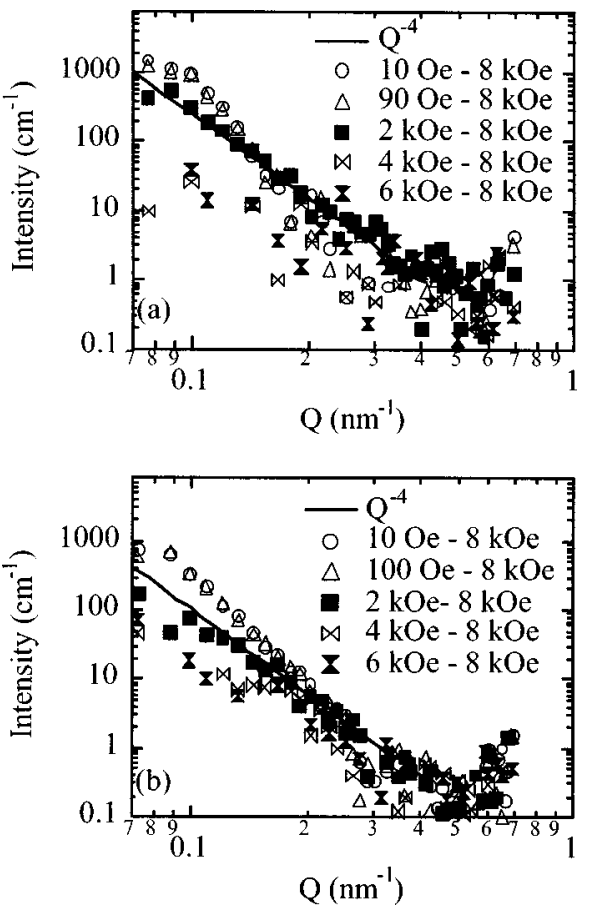

FIG. 10. Differential magnetic SANS from: (a) as-milled sample and (b) control sample annealed at $600{ }^{\circ} \mathrm{C}$. Profiles were obtained along directions perpendicular to the applied magnetic field and were processed by subtracting the profile obtained with an $8 \mathrm{kOe}$ field. Labels indicate the applied magnetic field in $\mathrm{kOe}$.

in Sec. IV B. From the square roots of the $G(0)$ for the as-milled material and the $G(0)$ for the material annealed at $265^{\circ} \mathrm{C}$, we obtain a $32 \%$ change in the magnetic scattering amplitude of the grain boundaries upon annealing. This is equivalent to an increase in magnetic moments in the grain boundary of $0.37 \mu_{B}$ /atom. This is a slightly larger change than obtained from the Mössbauer spectra, but agrees well with the grain boundary magnetic moments obtained in Sec. IV B.

\section{Magnetic saturation}

Minor differences were found in how the magnetic SANS from the nanocrystalline and control samples were affected by an increasing magnetic field. Figure 10 shows changes in the SANS caused by changes in the strength of the applied magnetic field. The profiles shown in Fig. 10 were obtained perpendicular to the direction of the applied field. These data therefore include the profile from the magnetic scattering under the condition of magnetic saturation. (When the applied magnetic field is small, magnetic scattering contrast occurs in all directions.) The data presented in Fig. 10 were obtained by subtracting the SANS profile from the corresponding profile obtained with an applied field of 8 kOe. Only a few data points from the $6 \mathrm{kOe}$ condition appear in Fig. 10, since similarity of the 6 and 8 kOe profiles caused many of the points in the difference to be negative, and therefore, absent from a logarithmic plot. The strongest magnetic contrast is found for the smallest applied fields. A wider range of magnetization orientations in the unsaturated material likely causes this. We believe this magnetic contrast in the unsaturated material originates with domain-like structures, which are eliminated as the material approaches saturation. The characteristic spatial size of these domain-like structures is probably on the order of $90 \mathrm{~nm}$ or larger, since we obtain the maximum change in magnetic contrast at our smallest value of $Q$. For $Q$ larger than $0.15 \mathrm{~nm}^{-1}$, the differential magnetic scattering shown in Fig. 10 is nearly the same for the as-milled sample and the large-grained control sample. For these large values of $Q$, the slope is approximately -4.0 . For $Q$ less than $0.15 \mathrm{~nm}^{-1}$, however, the slope of Fig. 10 is larger, being -6.8 for the as-milled material and -5.3 for the control sample. Previous measurements of differences in the magnetic SANS for saturated and unsaturated nanocrystals also had large slopes, ${ }^{1}$ but suitable control samples of large-grained material were not available for comparison. Such a large slope suggests that at low applied magnetic fields, the as-milled material may have a magnetic domain structure with a more regular periodicity than does the control sample. Such a claim should be confirmed by acquiring data at smaller values of $Q$, however. Finally, the as-milled data shows a larger change between 2 and $4 \mathrm{kOe}$, indicating that the magnetic saturation of the as-milled material occurs more quickly than for the control sample.

\section{CONCLUSIONS}

Three samples of $\mathrm{Ni}_{3} \mathrm{Fe}$ were prepared for study by Mössbauer spectrometry and SANS. These three materials were first characterized by dark field imaging with a TEM, and x-ray diffractometry lineshape analysis. The as-milled materials had the smallest average crystallite size, being about $15 \mathrm{~nm}$, whereas the material annealed at $265^{\circ} \mathrm{C}$ had a similar crystallite size, perhaps slightly larger at $20 \mathrm{~nm}$. The material annealed at $600{ }^{\circ} \mathrm{C}$ was shown by TEM to have much larger crystals of $300 \mathrm{~nm}$ or so. All materials had powder morphologies that were similar on the micron scale and larger.

Data from Mössbauer spectrometry and SANS measurements were interpreted by using comparable data sets from all samples, and using the material annealed at $600{ }^{\circ} \mathrm{C}$ as a "control", sample. From the nuclear SANS and the Mössbauer HMF distribution we found evidence for, as expected, a high density of grain boundaries in the as-milled material and the material annealed at $265^{\circ} \mathrm{C}$. The features of the nuclear scattering were similar for both samples, indicating little change of the atom density in grain boundaries upon annealing at $265^{\circ} \mathrm{C}$. The magnetic SANS was reduced significantly upon annealing, however, indicating that the magnetic moment density of the grain boundaries was increased by annealing at $265^{\circ} \mathrm{C}$. A similar type of change was found in the low field component of the HMF distribution. We attribute these changes in magnetic SANS and Mössbauer spectra to an increase in the average magnetic moment of grain boundaries. The Mössbauer spectra indicated an increase in grain boundary magnetic moments from $0.6 \mu_{B}$ /atom in the as-milled materials to $0.8 \mu_{B}$ /atom after annealing at $265^{\circ} \mathrm{C}$. (The magnetic moment per atom in large-grained $\mathrm{Ni}_{3} \mathrm{Fe}$ is $1.2 \mu_{B}$ /atom). Analysis of the scattering intensities and of the radial distribution functions derived 
from the SANS magnetic scattering curves showed that the grain boundary magnetic moments increased by $0.4 \mu_{B}$ /atom upon annealing at $265^{\circ} \mathrm{C}$. These changes in magnetic moment of the grain boundary of the $\mathrm{Ni}_{3} \mathrm{Fe}$ annealed at $265^{\circ} \mathrm{C}$ are probably caused by small changes in atom arrangements in the grain boundary region.

We measured changes in the magnetic SANS when an increasing magnetic field was applied to the sample. The SANS from the nanocrystalline samples and the control sample showed similar changes with applied field. We suggest that the domain structure of the nanocrystalline material may have a more regular spatial periodicity, but these structures are evidently of order $100 \mathrm{~nm}$ or larger, and our range in $Q$ was not optimal for their measurement.

\section{ACKNOWLEDGMENTS}

The Oak Ridge National Laboratory is managed for the Department of Energy by Lockheed Martin Energy Research, Oak Ridge, TN under Contract No. DE-AC0596OR22464. This work was supported by the U.S. Department of Energy under Contract No. DE-FG03-96ER45572.

${ }^{1}$ W. Wagner et al., J. Mater. Res. 6, 2305 (1991).

${ }^{2}$ M. Ohnuma et al., Physica B 213, 582 (1995).

${ }^{3}$ J. Kohlbrecher, A. Wiedenmann, and H. Wollenberger, Physica B 213, 579 (1995)

${ }^{4}$ P. G. Sanders et al., Scr. Metall. Mater. 29, 91 (1993).

${ }^{5}$ P. G. Sanders, J. R. Weertman, and J. G. Barker, J. Mater. Res. 11, 3110 (1996).

${ }^{6}$ A. J. Allen et al., Nanostruct. Mater. 7, 113 (1996).

${ }^{7}$ R. Schäfer et al., IEEE Trans. Magn. 32, 4383 (1996).
${ }^{8}$ W. Rave et al., IEEE Trans. Magn. 30, 4473 (1994).

${ }^{9}$ H. Gleiter, Prog. Mater. Sci. 33, 223 (1989).

${ }^{10}$ H. Gleiter, Nanostruct. Mater. 6, 3 (1995).

${ }^{11}$ B. Fultz, H. Kuwano, and H. Ouyang, J. Appl. Phys. 77, 3458 (1995).

${ }^{12}$ H. J. Fecht et al., Metall. Trans. A 21, 2333 (1990).

${ }^{13}$ J. Löffler and J. Weissmüller, Phys. Rev. B 52, 7076 (1995).

${ }^{14}$ T. Haubold et al., Phys. Lett. A 135, 461 (1989).

${ }^{15}$ M. Hirscher et al., J. Magn. Magn. Mater. 146, 117 (1995).

${ }^{16}$ F. R. N. Nabarro, Theory of Crystal Dislocations (Dover Publications Inc., New York, 1987).

${ }^{17}$ D. McLean, Grain Boundaries in Metals (Clarendon, Oxford, 1957).

${ }^{18}$ G. A. Chadwick and D. A. Smith, Grain Boundary Structure and Properties (Academic, New York, 1976).

${ }^{19}$ J. W. Edington, Practical Electron Microscopy in Materials Science (TechBooks, Herndon, Virginia, 1976).

${ }^{20}$ E. Scheil, Z. Metallkd. 27, 199 (1935)

${ }^{21}$ H. P. Klug and L. E. Alexander, X-Ray Diffraction Procedures (WileyInterscience, New York, 1974).

${ }^{22}$ G. E. Bacon, Neutron Diffraction (Oxford University Press, London, 1962).

${ }^{23}$ G. LeCaër and J. M. Dubois, J. Phys. E 12, 1083 (1979).

${ }^{24}$ J. Hesse, Hyperfine Interact. 47, 357 (1989).

${ }^{25}$ D. G. Rancourt and J. Y. Ping, Hyperfine Interact. 69, 497 (1991).

${ }^{26}$ J. Y. Ping, D. G. Rancourt, and R. A. Dunlap, J. Magn. Magn. Mater. 103, 285 (1992).

${ }^{27}$ S. J. Campbell and H. Gleiter, in Mössbauer Spectroscopy Applied to Magnetism and Materials Science, edited by G. Long and F. Gradjean (Plenum, New York, 1993), Vol. 1, pp. 241-303.

${ }^{28}$ B. Fultz, in Mössbauer Spectroscopy Applied to Magnetism and Materials Science, edited by G. Long and F. Gradjean (Plenum, New York, 1993), Vol. 1, pp. 1-31.

${ }^{29}$ J. W. Cable and E. O. Wollan, Phys. Rev. B 7, 2005 (1973).

${ }^{30}$ M. F. Collins and J. B. Forsyth, Philos. Mag. 8, 401 (1963).

${ }^{31}$ A. Heilmann and W. Zinn, Z. Metallkd. 58, 113 (1967).

${ }^{32}$ H. Ebert et al., J. Phys. F 18, 719 (1988).

${ }^{33}$ H. E. Schaefer et al., J. Less-Common Met. 140, 161 (1988). 